\title{
Local and nonlocal singular Liouville equations in Euclidean spaces
}

\author{
Ali Hyder* \\ UBC Vancouver \\ Gabriele Mancini* \\ ali.hyder@math .ubc.ca \\ Università di Padova \\ gabriele.mancini@math.unipd.it \\ Luca Martinazzi* \\ Università di Padova \\ luca.martinzzi@math.unipd.it
}

August 13, 2018

\begin{abstract}
We study metrics of constant $Q$-curvature in the Euclidean space with a prescribed singularity at the origin, namely solutions to the equation

$$
(-\Delta)^{\frac{n}{2}} w=e^{n w}-c \delta_{0} \text { on } \mathbb{R}^{n},
$$

under a finite volume condition. We analyze the asymptotic behaviour at infinity and the existence of solutions for every $n \geq 3$ also in a supercritical regime. Finally, we state some open problems.
\end{abstract}

\section{Introduction}

In this paper we will deal with the classification of the solutions to the equation

$$
(-\Delta)^{\frac{n}{2}} w=e^{n w}-c \delta_{0} \text { on } \mathbb{R}^{n},
$$

where $e^{n w} \in L^{1}\left(\mathbb{R}^{n}\right), c \in \mathbb{R}, \delta_{0}$ is a Dirac mass at the origin and $n \geq 2$. Equations of this kind arise naturally in the study of the prescribed $Q$-curvature problem with singularities. We recall that the $Q$-curvature is a curvature of order $n$ that was introduced by $[3,4,7]$ and in the last decades has been intensely studied in problems of conformal geometry. If $w$ is a solution of (1), then $e^{2 w}|d x|^{2}$ is a metric on $\mathbb{R}^{n}$ conformal to the Euclidean metric $|d x|^{2}$ and having $Q$-curvature equal to 1 everywhere except at the origin, where it has a special kind of singularity. When $n=2$, singularities of this kind are known as conical singularities and have been studied e.g. in $[2,20,21]$.

Writing

$$
w=u+\frac{c}{\gamma_{n}} \log |x|,
$$

\footnotetext{
*The authors are supported by the Swiss National Science Foundation projects n. PP00P2-144669, PP00P2170588/1 and P2BSP2-172064.
} 
we see that $u$ satisfies

$$
(-\Delta)^{\frac{n}{2}} u=|x|^{\frac{n c}{\gamma_{n}}} e^{n u} \text { on } \mathbb{R}^{n}
$$

where $\gamma_{n}:=\frac{(n-1) !}{2}\left|S^{n}\right|$ is such that

$$
(-\Delta)^{\frac{n}{2}}\left(\frac{1}{\gamma_{n}} \log \frac{1}{|x|}\right)=\delta_{0} \quad \text { in } \mathbb{R}^{n}
$$

and it will be convenient to study equation (2). More precisely, for a given $\alpha>-1$, we consider the problem

$$
\left\{\begin{array}{l}
(-\Delta)^{\frac{n}{2}} u=|x|^{n \alpha} e^{n u} \text { on } \mathbb{R}^{n}, \\
\Lambda:=\int_{\mathbb{R}^{n}}|x|^{n \alpha} e^{n u} d x<\infty .
\end{array}\right.
$$

Geometrically, if $u$ solves (3), then the metric $e^{2 u}|d x|^{2}$ has $Q$-curvature $|x|^{n \alpha}$ and total $Q$ curvature $\Lambda$. The parameter $\Lambda$ is also the volume of the metric $e^{2 w}|d x|^{2}$, and it plays a crucial role in the existence of solutions to (3).

In order to give a good definition of weak solutions to $(3)$, we need to define $(-\Delta)^{\frac{n}{2}}$ for a sufficiently large class of functions. Let $\mathcal{S}\left(\mathbb{R}^{n}\right)$ be the Schwartz space of rapidly decreasing functions, and for $s>0$ set

$$
L_{s}\left(\mathbb{R}^{n}\right):=\left\{w \in L_{\mathrm{loc}}^{1}\left(\mathbb{R}^{n}\right): \int_{\mathbb{R}^{n}} \frac{|w(x)|}{1+|x|^{n+2 s}} d x<\infty\right\} .
$$

Given a function $v \in L_{s}\left(\mathbb{R}^{n}\right), s>0$, we define $(-\Delta)^{s} v$ as a tempered distribution as follows:

$$
\left\langle(-\Delta)^{s} v, \varphi\right\rangle:=\int_{\mathbb{R}^{n}} v(-\Delta)^{s} \varphi d x \quad \text { for every } \varphi \in \mathcal{S}\left(\mathbb{R}^{n}\right),
$$

where

$$
(-\Delta)^{\frac{s}{2}} \varphi:=\mathcal{F}^{-1}\left(|\xi|^{s} \mathcal{F} \varphi(\xi)\right)
$$

and

$$
\mathcal{F} \varphi(\xi):=\frac{1}{(2 \pi)^{\frac{n}{2}}} \int_{\mathbb{R}^{n}} \varphi(x) e^{-i x \cdot \xi} d x
$$

is the Fourier transform. The right-hand side of (4) makes sense because

$$
(-\Delta)^{s} \varphi(x)=O\left(|x|^{-n-2 s}\right) \quad \text { as }|x| \rightarrow \infty,
$$

see e.g. [14, Proposition 2.1].

Definition 1.1. Given $m \geq 1$ and a tempered distribution $f \in \mathcal{S}^{\prime}\left(\mathbb{R}^{n}\right)$, we say that $u$ is a solution of

$$
(-\Delta)^{\frac{m}{2}} u=f \quad \text { in } \mathbb{R}^{n}
$$

if the following holds. In case $m \geq 2$ even we require $u \in L_{\mathrm{loc}}^{1}\left(\mathbb{R}^{n}\right)$ and

$$
\int_{\mathbb{R}^{n}} u(-\Delta)^{\frac{m}{2}} \varphi d x=\langle f, \varphi\rangle \quad \text { for every } \varphi \in C_{c}^{\infty}\left(\mathbb{R}^{n}\right) .
$$

In case $m \geq 1$ odd we require $u \in L_{\frac{m}{2}}\left(\mathbb{R}^{n}\right)$ and

$$
\int_{\mathbb{R}^{n}} u(-\Delta)^{\frac{m}{2}} \varphi d x=\langle f, \varphi\rangle \quad \text { for every } \varphi \in \mathcal{S}\left(\mathbb{R}^{n}\right) .
$$


As we shall see in Theorem 2.1, weak solutions to (3) are smooth away from the origin and at least $C^{0}$ near the origin.

In the classical case $n=2$, for any $\alpha>-1$ there are explicit solutions to (3) taking the form

$$
u(x)=\log \left(\frac{2(\alpha+1)}{1+|x|^{2(\alpha+1)}}\right), \quad \Lambda=4 \pi(\alpha+1) .
$$

With the scaling $u_{\lambda}(x)=u\left(\lambda^{\frac{1}{1+\alpha}} x\right)+\log \lambda$ we obtain the family of solutions

$$
u(x)=\log \left(\frac{2(\alpha+1) \lambda}{1+\lambda^{2}|x|^{2(\alpha+1)}}\right), \quad \Lambda=4 \pi(\alpha+1), \quad \lambda>0, \alpha>-1 .
$$

In fact, as shown by Chen-Li [6], when $n=2$ for every solution to (3) we necessarily have $\Lambda=4 \pi(1+\alpha)$. In [20] Prajapat-Tarantello proved that solutions as in (5) exhaust the set of solutions of (3) if $\alpha$ is not an integer, while when $\alpha \in \mathbb{N}$ instead there exist more solutions, all of which can be written using the complex notation in the form

$$
u(z)=\log \left(\frac{2(\alpha+1) \lambda}{1+\lambda^{2}\left|z^{\alpha+1}-\zeta\right|^{2}}\right), \quad \lambda>0, \zeta \in \mathbb{C} .
$$

Interestingly the solutions in (6) are not radially symmetric, except in the case $\zeta=0$ (when they reduce to (5)) or $\alpha=0$ (in which case they are radial about the point $\zeta$ ) and blow up at the $\alpha+1$ roots of the complex equation $z^{\alpha+1}=\zeta$ as $\lambda \rightarrow \infty$.

When $n>2$, the structure of the set of solutions of (3) becomes richer. If $\alpha=0$ one can still identify a special family of solutions, known as normal (cfr. Definition 1.2) or sperical solutions, having an explicit expression similar to (6) (with $\alpha=0$ ). These solutions have total $Q$-curvature $\Lambda=\Lambda_{1}:=(n-1) !\left|S^{n}\right|$ and are the only solutions of (3) with $u=o\left(|x|^{2}\right)$ as $|x| \rightarrow+\infty$. However, in addition to these solutions there are also non-normal solutions, behaving polynomially at infinity and with $\Lambda$ not necessarily equal to $\Lambda_{1}$ see e.g. [5, 12, 15, 16, 23].

When $n \neq 2$ and $\alpha \neq 0$ we do not have explicit formulas for solutions to (3) (not even when $\alpha$ is integer, to the best of our knowledge) so that the existence of even one single solution is not obvious. We shall prove two existence results based on a Schauder fixed-point argument, in a spirit similar to that of previous works, such as $[12,13,15,23]$.

There are two crucial ingredients in this approach. One is to have a good ansatz, namely restrict the set among which we will look for solutions to functions having a particular asymptotic behaviour. The second ingredient is a precise information on the value of $\Lambda$ for normal solutions to (3), namely solutions to an integral equation, see Definition 1.2. Both this properties are contained in the following theorem, which extends to the case $\alpha \neq 0$ the classification results in $[17,18,22,16,14]$.

Theorem 1.1. For $\alpha>-1$ let $u$ solve (3) and define $v$ as follows

$$
v(x)=\frac{1}{\gamma_{n}} \int_{\mathbb{R}^{n}} \log \left(\frac{1+|y|}{|x-y|}\right)|y|^{n \alpha} e^{n u(y)} d y .
$$

Then there exists an upper-bounded polynomial $p$ of degree at most $n-1$ such that $u=v+p$. Moreover

$$
\lim _{|x| \rightarrow \infty} \frac{v(x)}{\log |x|}=-\beta:=-\frac{\Lambda}{\gamma_{n}} .
$$

Finally, if $p$ is constant, then $\Lambda=\Lambda_{1}(1+\alpha)$. 
Notice that $p$ being upper bounded implies that $p$ has even degree, hence $p$ has degree at most $n-2$ when $n$ is even.

Definition 1.2 (Normal solutions). We call a solution $u$ to (3) normal if writing $u=v+p$ as in Theorem 1.1 the polynomial $p$ is constant. Equivalently if there exists a constant $c \in \mathbb{R}$ such that $u$ solves the integral equation

$$
u(x)=\frac{1}{\gamma_{n}} \int_{\mathbb{R}^{n}} \log \left(\frac{1+|y|}{|x-y|}\right)|y|^{n \alpha} e^{n u(y)} d y+c .
$$

The proof of Theorem 1.1 is innovative in comparison to the previous works, which it also simplifies considerably (see Remark 3.1). It is based on a Campanato-space estimate (Lemma 3.6). In fact, instead of having an $L^{\infty}$-upper bound on the function $v$, which is usually difficult to obtain in the case $\alpha=0$ and appears to be much more challenging in the case $\alpha \neq 0$, we content ourselves with a decay estimate on the oscillation of $v$, which will be sufficient to conclude.

We can summarize Theorem 1.1 by saying that all solutions to (3) have the form $v+p$, where $v$ behaves logarithmically at infinity and $p$ is a polynomial, and if $p$ is constant, then $\Lambda=\Lambda_{1}(1+\alpha)$. Using this information we can move towards the existence results.

In the first result we show existence of radial solutions up to the critical treshold $\Lambda_{1}(1+\alpha)$.

Theorem 1.2. Let $n \geq 3$ and $\alpha>-1$. Then for every $0<\Lambda<\Lambda_{1}(1+\alpha)$ there exists a radially symmetric solution to (3). For $\Lambda=\Lambda_{1}(1+\alpha)$ there exists a radially symmetric and normal solution to $(3)$.

Existence for every $\Lambda<\Lambda_{1}(1+\alpha)$ is obtained by a standard compactness argument. These solutions will have the form $v+p$ with $p(x)=-|x|^{2}$. The normal solutions obtained for the case $\Lambda=\Lambda_{1}(1+\alpha)$ are the higher-dimensional equivalent of (5), whose existence is not obvious, since we do not have explicit formulas for them. For this part we will use a blow-up argument together with a non-existence result based on a Pohozaev-type identity.

Next we show that breaking radial symmetry, we are able to produce solutions to (3) above the critical threshold.

Theorem 1.3. Let $n \geq 3$. Then for every $\alpha>-1$ and for every $\Lambda \in\left(0, \Lambda_{1}\right)$ there exists a solution to (3).

Notice that $\Lambda_{1}>\Lambda_{1}(1+\alpha)$ for $\alpha \in(-1,0)$ in Theorem 1.3, hence we have an existence result above the critical threshold of compactness.

As we shall see, the solutions given by Theorem 1.3 are non-radial, by construction. On the other hand, in dimension 3 and 4 this is also a necessity. Indeed from the Pohozaev identity we obtain:

Proposition 1.1. For $n=3$ and $n=4$ every radial solution $u$ to (3) satisfies $\Lambda \leq \Lambda_{1}(1+\alpha)$ with identity if and only if $u$ is normal.

\subsection{Some open problems}

Using a variational argument as in [12] one should be able to find solutions to (3) of the form $u=v+p$ with the polynomial $p$ prescribed. For instance one could try to prove that for $n \geq 3$, $\alpha>-1,0<\Lambda<\Lambda_{1} \min \{1,1+\alpha\}$ and a given polynomial $p$ with $\operatorname{deg}(p) \leq n-1$ and satisfying

$$
p(x) \stackrel{|x| \rightarrow \infty}{\longrightarrow}-\infty,
$$


there exists a solution $u$ to (3) such that

$$
u(x)=p(x)-\frac{2 \Lambda}{\Lambda_{1}} \log |x|+C+o(1), \quad o(1) \stackrel{|x| \rightarrow \infty}{\longrightarrow} 0 .
$$

Also the existence of solutions to (3) for arbitrarily large $\Lambda$ is open, in analogy with the case $\alpha=0$ studied in $[11,13,19]$. In the radial case, using methods from [13] it should be possible to prove that for $n \geq 5, \alpha>-1$ and for every $\Lambda>0$ there exists a radially symmetric solution to (3). Notice that the condition $n \geq 5$ is necessary in view of Proposition 1.1 and the known results in dimension 1 and 2 . If instead we drop the radial symmetry, it is open whether already in dimension 3 and 4 we can have solutions to (3) with arbitrarily large $\Lambda$, for $\alpha \neq 0$.

Notation In what follows $B_{R}(x)$ will denote the ball of radius $R$ centered at $x$ (the dependence on $x$ will often be omitted if $x=0$ ) and $C$ will denote a generic constant that can change from line to line.

Acknowledgements During his $\mathrm{PhD}$ at the University of Basel under the guidance of the third author, Stefano Iula collaborated with the first and second authors to the proof of Theorem 1.1 .

\section{Regularity of solutions}

If $\Omega \subseteq \mathbb{R}^{n}$ is an open set, we denote

$$
C^{s}(\Omega):=\left\{u \in C^{\lfloor s\rfloor}(\Omega): D^{\lfloor s\rfloor} u \in C^{0, s-\lfloor s\rfloor}(\Omega)\right\}, \quad s-1<\lfloor s\rfloor \leq s,\lfloor s\rfloor \in \mathbb{N},
$$

Then, we set

$$
C_{\mathrm{loc}}^{s}\left(\mathbb{R}^{n}\right):=\left\{u \in C^{0}\left(\mathbb{R}^{n}\right):\left.u\right|_{\Omega} \in C^{s}(\Omega) \text { for every } \Omega \Subset \mathbb{R}^{n}\right\} .
$$

Theorem 2.1. Let $u$ be a solution of (3) with $\alpha>-1$. If $\alpha \in(-1,0)$ or $n \alpha-1 \in 2 \mathbb{N}$, then $u \in C^{\infty}\left(\mathbb{R}^{n} \backslash\{0\}\right) \cap C_{\text {loc }}^{s}\left(\mathbb{R}^{n}\right)$, for $s<n(1+\alpha)$. If $\alpha>0$ with n $\alpha \notin \mathbb{N}$ we have $u \in$ $C^{\infty}\left(\mathbb{R}^{n} \backslash\{0\}\right) \cap C_{\mathrm{loc}}^{n(1+\alpha)}\left(\mathbb{R}^{n}\right)$, and if $n \alpha \in 2 \mathbb{N}$ we have $u \in C^{\infty}\left(\mathbb{R}^{n}\right)$.

Proof. First, we claim that $e^{n u} \in L_{\text {loc }}^{q}\left(\mathbb{R}^{n}\right)$ for any $q \geq 1$. Indeed, given any $q$, we can take $\varepsilon=\varepsilon(q)$ such that $q<\frac{\gamma_{n}}{\varepsilon}$ and we can split $|x|^{n \alpha} e^{n u}=f_{1}+f_{2}$, where $f_{1}, f_{2} \geq 0$ and

$$
f_{1} \in L^{1}\left(\mathbb{R}^{n}\right) \cap L^{\infty}\left(\mathbb{R}^{n}\right), \quad\left\|f_{2}\right\|_{L^{1}\left(\mathbb{R}^{n}\right)} \leq \varepsilon
$$

Let us define the functions

$$
u_{i}(x):=\frac{1}{\gamma_{n}} \int_{\mathbb{R}^{n}} \log \left(\frac{1+|y|}{|x-y|}\right) f_{i}(y) d y, \quad x \in \mathbb{R}^{n}, i=1,2,
$$

and

$$
u_{3}:=u-u_{1}-u_{2} .
$$


It is easy to see that $u_{1} \in C^{n-1}\left(\mathbb{R}^{n}\right)$ and that $u_{3}$ is $\frac{n}{2}$-harmonic and hence $u_{3} \in C^{\infty}\left(\mathbb{R}^{n}\right)$. Moreover, using Jensen's inequality we get

$$
\begin{aligned}
\int_{B_{R}} e^{n q u_{2}} d x & =\int_{B_{R}} \exp \left(\int_{\mathbb{R}^{n}} \frac{n q\left\|f_{2}\right\|}{\gamma_{n}} \log \left(\frac{1+|y|}{|x-y|}\right) \frac{f_{2}(y)}{\left\|f_{2}\right\|} d y\right) d x \\
& \leq \int_{B_{R}} \int_{\mathbb{R}^{n}} \exp \left(\frac{n q\left\|f_{2}\right\|}{\gamma_{n}} \log \left(\frac{1+|y|}{|x-y|}\right)\right) \frac{f_{2}(y)}{\left\|f_{2}\right\|} d y d x \\
& =\frac{1}{\left\|f_{2}\right\|} \int_{\mathbb{R}^{n}} f_{2}(y) \int_{B_{R}}\left(\frac{1+|y|}{|x-y|}\right)^{\frac{n q\left\|f_{2}\right\|}{\gamma_{n}}} d x d y \\
& \leq C(q, R)
\end{aligned}
$$

where $\|\cdot\|$ denotes the $L^{1}\left(\mathbb{R}^{n}\right)$ norm. Hence the claim is proved.

Set $\bar{p}:=-\frac{1}{\alpha}$ for $\alpha \in(-1,0)$ and $\bar{p}:=\infty$ if $\alpha>0$. Then $|\cdot|^{n \alpha} \in L_{\text {loc }}^{p}\left(\mathbb{R}^{n}\right)$ for every $1 \leq p<\bar{p}$. It follows that $(-\Delta)^{\frac{n}{2}} u=|\cdot|^{n \alpha} e^{n u} \in L_{\text {loc }}^{p}\left(\mathbb{R}^{n}\right)$ for $p<\bar{p}$, and this implies $u \in W_{\text {loc }}^{n, p}\left(\mathbb{R}^{n}\right)$ for $p<\bar{p}$. Indeed, for any given $R>0$, we can write $u=v_{1}+v_{2}+v_{3}$, where $v_{3}$ is $\frac{n}{2}$-harmonic (and thus $v_{3} \in C^{\infty}\left(\mathbb{R}^{n}\right)$ ) and

$$
v_{i}(x):=\frac{1}{\gamma_{n}} \int_{\mathbb{R}^{n}} \log \left(\frac{1+|y|}{|x-y|}\right) g_{i}(y) d y, \quad x \in \mathbb{R}^{n}, i=1,2,
$$

with

$$
g_{1}=|\cdot|^{n \alpha} e^{n u} \chi_{B_{R}}, \quad g_{2}=|\cdot|^{n \alpha} e^{n u} \chi_{B_{R}^{c}} .
$$

Differentiating (11) we obtain that $v_{1} \in W^{n, p}\left(B_{\frac{R}{2}}\right)$ (by the Calderon-Zygmund theory) and $v_{2} \in C^{\infty}\left(B_{\frac{R}{2}}\right)$.

By the Sobolev embedding we then infer that $u \in C_{\mathrm{loc}}^{s}\left(\mathbb{R}^{n}\right)$ for $s<n(1+\min \{\alpha, 0\})$.

Since $|\cdot|^{n \alpha} \in C^{\infty}\left(\mathbb{R}^{n} \backslash\{0\}\right)$, by bootstrapping regularity we see that $u \in C^{\infty}\left(\mathbb{R}^{n} \backslash\{0\}\right)$ for every $\alpha>-1$. Now, if $\alpha \geq 0$, we observe that

$$
|\cdot|^{n \alpha} \in \begin{cases}C^{n \alpha}\left(\mathbb{R}^{n}\right) & \text { if } n \alpha \notin \mathbb{N}, \\ C_{\mathrm{loc}}^{s}\left(\mathbb{R}^{n}\right) & \text { if } s<n \alpha, n \alpha-1 \in 2 \mathbb{N}, \\ C^{\infty}\left(\mathbb{R}^{n}\right) & \text { if } n \alpha \in 2 \mathbb{N} .\end{cases}
$$

In any case, we can conclude the proof by bootstrapping regularity using Schauder estimates.

\section{Proof of Theorem 1.1}

Lemma 3.1. Let $u$ be a solution to Problem (3) and $v$ as in (7). Then for $|x| \geq 1$ we have

$$
v(x) \geq-\beta \log |x|
$$

where $\beta$ is as in (8).

Proof. Since $|x| \geq 1$, thanks to the triangular inequality we have

$$
|x-y| \leq|x|+|y| \leq|x|+|x||y|=|x|(1+|y|),
$$


for any $y \in \mathbb{R}^{n}$. Therefore

$$
\log \left(\frac{1+|y|}{|x-y|}\right) \geq-\log |x|
$$

Lemma 3.2. Let $u$ be a solution to Problem (3) and $v$ as in (7). Then $u=v+p$ where $p$ is $a$ polynomial of degree at most $n-1$.

Proof. Set $p=u-v$ so that $(-\Delta)^{\frac{n}{2}} p=0$. From Lemma 3.1 we have

$$
p(x) \leq u(x)+\beta \log (1+|x|)+C .
$$

Recalling that $|\cdot|^{n \alpha} e^{n u} \in L^{1}$, it follows from a Liouville-type theorem (see Theorem A.1 in the Appendix) that $p$ is a polynomial of degree at most $n-1$.

Lemma 3.3. Let $p$ be a polynomial as in Lemma 3.2. Then

$$
\sup _{x \in \mathbb{R}^{n}} p(x)<+\infty
$$

In particular $p$ has even degree. Moreover for every $q \geq 1$ and $0<\rho \leq \rho_{0}$ there exists $C=$ $C\left(q, \rho_{0}\right)$ such that

$$
\int_{B_{\rho}(x)} e^{q p} d x \leq C|x|^{n(\beta-\alpha)}
$$

for any $x \in \mathbb{R}^{n}$ with $|x| \geq 1$.

Proof. We start by proving (13). Following [18] we define

$$
f(r)=\sup _{\partial B_{r}} p
$$

From Theorem 3.1 in [10] it follows that if $\sup _{\mathbb{R}^{n}} p=+\infty$ then there exists $s>0$ such that

$$
\lim _{r \rightarrow+\infty} \frac{f(r)}{r^{s}}=+\infty
$$

From Lemma $3.2 p$ is a polynomial of degree at most $n-1$. In particular, we have that $|\nabla p(x)| \leq C|x|^{n-2}$ for $|x|$ large. From Lemma 3.1 it follows that there exists $R>0$ such that for every $r \geq R$ we can find $x_{r}$ with $\left|x_{r}\right|=r$ such that

$$
u(y)=v(y)+p(y) \geq r^{s}
$$

for $\left|y-x_{r}\right| \leq \frac{1}{r^{n-2}}$. Now using Fubini we get

$$
\begin{aligned}
\int_{\mathbb{R}^{n}}|x|^{n \alpha} e^{n u} d x & \geq \int_{R}^{+\infty} \int_{\partial B_{r}(0) \cap B_{r^{2-n}}\left(x_{r}\right)} r^{n \alpha} e^{n r^{s}} d \sigma d r \\
& \geq C \int_{R}^{+\infty} \frac{r^{n \alpha} e^{n r^{s}}}{r^{(n-2)(n-1)}} d r=+\infty
\end{aligned}
$$


that is a contradiction, hence (13) is proven.

The proof of (14) follows at once from (13) and Lemma 3.1. Indeed, if $|x| \geq 2 \rho_{0}$ then

$$
C \geq \int_{B_{\rho}(x)}|y|^{n \alpha} e^{n u(y)} d y \geq C \int_{B_{\rho}(x)}|y|^{n(\alpha-\beta)} e^{n p(y)} d y \geq C|x|^{n(\alpha-\beta)} \int_{B_{\rho}(x)} e^{q p(y)} d y,
$$

while (14) is trivial for $|x| \in\left[1,2 \rho_{0}\right)$.

Lemma 3.4. For any $\varepsilon>0$ there exists $R>0$ such that for $|x| \geq R$

$$
v(x) \leq(-\beta+\varepsilon) \log |x|+\frac{1}{\gamma_{n}} \int_{B_{1}(x)} \log \left(\frac{1}{|x-y|}\right)|y|^{n \alpha} e^{n u} d y .
$$

Proof. The simple proof is similar to [17, pag. 213] and is omitted.

Lemma 3.5. For any $q \geq 1, \varepsilon_{1}, \varepsilon_{2}>0$ there exists a constant $C=C\left(q, \varepsilon_{1}, \varepsilon_{2}\right)$ such that for $0<\rho \leq 1$ and $x \in \mathbb{R}^{n}$

$$
\frac{1}{\rho^{n-\varepsilon_{2}}} \int_{B_{\rho}(x)} e^{q v(y)} d y \leq \frac{C}{|x|^{\left(\beta-\varepsilon_{1}\right) q}} .
$$

Proof. For $x$ in a compact set the statement is trivial. Set $f(x)=|x|^{n \alpha} e^{n u(x)}$, and fix $R>0$ such that

$$
\frac{q}{\gamma_{n}}\|f\|_{L^{1}\left(B_{R}^{c}\right)} \leq \varepsilon_{2} .
$$

From Lemma 3.4 up to taking $R$ larger, we have for $|z|>R+1$

$$
\begin{aligned}
v(z) & \leq\left(-\beta+\varepsilon_{1}\right) \log |z|+\frac{1}{\gamma_{n}} \int_{B_{1}(z)} \log \left(\frac{1}{|z-y|}\right) f(y) \chi_{|z-y| \leq 1} d y \\
& \leq\left(-\beta+\varepsilon_{1}\right) \log |z|+\frac{1}{\gamma_{n}} \int_{B_{R}^{c}(0)} \log \left(\frac{1}{|z-y|}\right) f(y) \chi_{|z-y| \leq 1} d y .
\end{aligned}
$$

Applying Jensen inequality with respect to the measure $d \mu(y):=\frac{f(y)}{\|f\|_{L^{1}\left(B_{R}^{c}\right)}} d y$ in $B_{R}^{c}$, we get for $|x|>R+2$

$$
\begin{aligned}
\int_{B_{\rho}(x)} e^{q v(z)} d z & \leq \int_{B_{\rho}(x)} \frac{1}{|z|^{\left(\beta-\varepsilon_{1}\right) q}} e^{q \frac{\|f\|_{L^{1}\left(B_{R}^{c}\right)}}{\gamma_{n}} \int_{B_{R}^{c}(0)} \log \left(\frac{1}{|z-y|}\right) \chi_{|z-y| \leq 1} d \mu(y)} d z \\
& \leq \frac{C}{|x|^{\left(\beta-\varepsilon_{1}\right) q}} \int_{B_{\rho}(x)} \int_{B_{R}^{c}(0)} e^{q \frac{\|f\|_{L^{1}\left(B_{R}^{c}\right)}}{\gamma_{n}} \log \left(\frac{1}{|z-y|}\right) \chi_{|z-y| \leq 1}} d \mu(y) d z \\
& \leq \frac{C}{|x|^{\left(\beta-\varepsilon_{1}\right) q}} \int_{B_{R}^{c}(0)} \int_{B_{\rho}(x)} e^{\varepsilon_{2} \log \left(\frac{1}{|z-y|}\right) \chi_{|z-y| \leq 1}} d z d \mu(y) \\
& \leq \frac{C}{|x|^{\left(\beta-\varepsilon_{1}\right) q}} \int_{B_{R}^{c}(0)} \int_{B_{\rho}(x)}\left(1+\frac{1}{|z-y|^{\varepsilon_{2}}}\right) d z d \mu(y) \\
& \leq \frac{C \rho^{n-\varepsilon_{2}}}{|x|^{\left(\beta-\varepsilon_{1}\right) q}}
\end{aligned}
$$


Remark 3.1. Using Lemma 3.5 one gets a simpler proof of Theorem 1.1 in the classical case $\alpha=0$, or even if $\alpha \in(-1, \beta)$. Indeed using the Hölder inequality in (15), with $\varepsilon \leq \beta-\alpha$ and applying Lemma 3.5 with $\varepsilon_{1}=\varepsilon$ and $\rho=1$ we get for $|x|$ large

$$
\int_{B_{1}(x)} \log \left(\frac{1}{|x-y|}\right)|y|^{n \alpha} e^{n u} d y \leq C|x|^{n \alpha} \int_{B_{1}(x)} \log \left(\frac{1}{|x-y|}\right) e^{n v} d y \leq C \frac{|x|^{n \alpha}}{|x|^{n(\beta-\varepsilon)}} \leq C .
$$

The proof of (8) follows at once from (15), and the rest of the proof will follow easily from the Pohozaev identity as we shall see below.

Remark 3.2. Arguing as in Lemmas 3.1-3.5 one can also obtain the following: Let $v$ be a solution to

$$
v(x)=\frac{1}{\gamma_{n}} \int_{\mathbb{R}^{n}} \log \left(\frac{1+|y|}{|x-y|}\right) K(y) e^{n v(y)} d y+c
$$

for some $c \in \mathbb{R}$ and some non-negative function $K \in L^{\infty}\left(\mathbb{R}^{n} \backslash B_{1}\right)$ with $K e^{n v} \in L^{1}\left(\mathbb{R}^{n}\right)$. Then

$$
\lim _{|x| \rightarrow \infty} \frac{v(x)}{\log |x|}=-\frac{1}{\gamma_{n}} \int_{\mathbb{R}^{n}} K e^{n v} d x
$$

From now on we shall assume $\alpha \geq \beta$.

Lemma 3.6. We have

$$
\tau(x):=\sup _{\rho \in(0,4]} \frac{1}{\rho^{n+\frac{1}{\log |x|}}} \int_{B_{\rho}(x)}\left|v(y)-f_{B_{\rho}(x)} v(z) d z\right| d y=o(1),
$$

with $o(1) \rightarrow 0$ as $|x| \rightarrow \infty$. As a consequence we have

$$
[v]_{C^{0, \frac{1}{\log \left(\left|x_{0}\right|+1\right)}}\left(B_{1}\left(x_{0}\right)\right)}:=\sup _{\substack{x, y \in B_{1}\left(x_{0}\right) \\ x \neq y}} \frac{|v(x)-v(y)|}{|x-y|^{\frac{1}{\log \left(\left|x x_{0}\right|+1\right)}}}=o(1) \log \left(\left|x_{0}\right|+1\right),
$$

with $o(1) \rightarrow 0$ as $\left|x_{0}\right| \rightarrow \infty$.

Proof. We start proving (16). We have

$$
\int_{B_{\rho}(x)} f_{B_{\rho}(x)}|v(y)-v(z)| d z d y \leq \int_{B_{\rho}(x)} f_{B_{\rho}(x)} \frac{1}{\gamma_{n}} \int_{\mathbb{R}^{n}}\left|\log \left(\frac{|z-\xi|}{|y-\xi|}\right)\right| f(\xi) d \xi d z d y,
$$

where $f(x):=|x|^{n \alpha} e^{n u(x)}$. By (14), Lemma 3.5 and Hölder's inequality we get for given $\varepsilon_{1}, \varepsilon_{2}, r>0$, that

$$
\begin{aligned}
\int_{B_{r}(x)} f(y) d y & \leq C|x|^{n \alpha}\left(\int_{B_{r}(x)} e^{2 n v} d y\right)^{\frac{1}{2}}\left(\int_{B_{r}(x)} e^{2 n p} d y\right)^{\frac{1}{2}} \\
& \leq C|x|^{n \alpha}\left(\frac{r^{n-\varepsilon_{2}}}{|x|^{2 n\left(\beta-\varepsilon_{1}\right)}}\right)^{\frac{1}{2}}\left(|x|^{n(\beta-\alpha)}\right)^{\frac{1}{2}} \\
& =C|x|^{c_{1}} r^{\frac{n}{2}-\frac{\varepsilon_{2}}{2}}, \quad c_{1}:=\frac{n(\alpha-\beta)}{2}+n \varepsilon_{1} .
\end{aligned}
$$


Choosing $r=2 \sqrt{\rho}$, together with Lemma A.2 this yields

$$
\begin{aligned}
(I) & :=\int_{B_{\rho}(x)} f_{B_{\rho}(x)} \int_{B_{2 \sqrt{\rho}}(x)}\left|\log \left(\frac{|z-\xi|}{|y-\xi|}\right)\right| f(\xi) d \xi d z d y \\
& \leq C \rho^{n} \int_{B_{2 \sqrt{\rho}(x)}} f(\xi) d \xi \\
& \leq C \rho^{\frac{5 n}{4}-\frac{\varepsilon_{2}}{4}|x|^{c_{1}}} .
\end{aligned}
$$

Now choosing $\varepsilon_{2} \leq \frac{n}{4}$ and taking $|x|$ sufficiently large, we have $\frac{n}{4}-\frac{\varepsilon_{2}}{4}-\frac{1}{\log |x|}>\frac{n}{8}$. Then, for $\rho \in\left(0,|x|^{-\frac{8 c_{1}}{n}}\right)$, we further bound

$$
\begin{aligned}
(I) & \leq C \rho^{n+\frac{1}{\log |x|}} \rho^{\frac{n}{4}-\frac{\varepsilon_{2}}{4}-\frac{1}{\log |x|}}|x|^{c_{1}} \\
& =o(1) \rho^{n+\frac{1}{\log |x|}} \rho^{\frac{n}{8}}|x|^{c_{1}} \\
& =o(1) \rho^{n+\frac{1}{\log |x|}},
\end{aligned}
$$

and with Lemma A.2 part $i i)$

$$
(I I):=\int_{B_{\rho}(x)} f_{B_{\rho}(x)} \int_{B_{2 \sqrt{\rho}}(x)^{c}}\left|\log \left(\frac{|z-\xi|}{|y-\xi|}\right)\right| f(\xi) d \xi d z d y \leq C \rho^{n+\frac{1}{2}}=o(1) \rho^{n+\frac{1}{\log |x|}} .
$$

For $\rho \in\left(|x|^{-\frac{8 c_{1}}{n}}, 1\right)$ we write

$$
\int_{B_{\rho}(x)} f_{B_{\rho}(x)} \int_{\mathbb{R}^{n}}\left|\log \left(\frac{|z-\xi|}{|y-\xi|}\right)\right| f(\xi) d \xi d z d y=I_{1}+I_{2},
$$

where

$$
I_{i}:=\int_{B_{\rho}(x)} f_{B_{\rho}(x)} \int_{A_{i}}\left|\log \left(\frac{|z-\xi|}{|y-\xi|}\right)\right| f(\xi) d \xi d z d y, \quad A_{1}:=B_{\frac{|x|}{2}}(x), A_{2}=A_{1}^{c} .
$$

Using Lemma A.2 we bound

$$
I_{1} \leq C \rho^{n} \int_{A_{1}} f(\xi) d \xi=o(1) \rho^{n}=o(1) \rho^{n+\frac{1}{\log |x|}},
$$

and

$$
I_{2}=o(1) \rho^{n}=o(1) \rho^{n+\frac{1}{\log |x|}}
$$

where we have used that

$$
\|f\|_{L^{1}\left(A_{1}\right)} \stackrel{|x| \rightarrow \infty}{\longrightarrow} 0, \quad|x|^{\frac{1}{\log |x|}}=e, \quad \operatorname{dist}\left(A_{2}, x\right) \stackrel{|x| \rightarrow \infty}{\longrightarrow} \infty .
$$

This proves (16).

For the proof of (17) we essentially follow Theorem 5.5 of [8]. Given $x \in \mathbb{R}^{n}$ and $\rho>0$ we use the notation

$$
v_{x, \rho}:=f_{B_{\rho}(x)} v(y) d y
$$


Fix

$$
\sigma=\frac{1}{\log \left(\left|x_{0}\right|+1\right)} \in(0,1) \quad \text { and } \quad \lambda=n+\sigma .
$$

For $0<r<R \leq 4, x \in B_{1}\left(x_{0}\right)$ and $z \in B_{R}(x)$ we have

$$
\left|v_{x, r}-v_{x, R}\right| \leq\left|v(z)-v_{x, r}\right|+\left|v(z)-v_{x, R}\right|,
$$

and integrating with respect to $z$ we bound

$$
\begin{aligned}
\left|v_{x, R}-v_{x, r}\right| & \leq \frac{1}{\left|B_{r}\right|}\left(\int_{B_{r}(x)}\left|v(z)-v_{x, r}\right| d z+\int_{B_{R}(x)}\left|v(z)-v_{x, R}\right| d z\right) \\
& \leq \frac{C}{r^{n}}\left(r^{\lambda}+R^{\lambda}\right) \tau(x) \\
& \leq C R^{\lambda} r^{-n} \tau(x) .
\end{aligned}
$$

Setting $R_{k}=\frac{R}{2^{k}}$ we infer

$$
\left|v_{x, R_{k}}-v_{x, R_{k+1}}\right| \leq C R^{\sigma} 2^{-k \sigma} \tau(x) .
$$

Applying the triangular inequality for $h>k$ we bound

$$
\left|v_{x, R_{h}}-v_{x, R_{k}}\right| \leq \sum_{j=k}^{h-1}\left|v_{x, R_{j+1}}-v_{x, R_{j}}\right| \leq C R^{\sigma} \sum_{j=k}^{h-1} 2^{-j \sigma} \tau(x) \leq \frac{C R^{\sigma}}{1-2^{-\sigma}} \tau(x) .
$$

Since the function $s \mapsto \frac{s}{1-2^{-s}}$ is increasing in $[0,1]$ one has $\frac{1}{1-2^{-\sigma}} \leq \frac{2}{\sigma}$ and we get

$$
\left|v_{x, R_{h}}-v_{x, R_{k}}\right| \leq \frac{C}{\sigma} R^{\sigma} \tau(x) \quad 0 \leq k<h .
$$

Taking $k=0$ and letting $h \rightarrow \infty$ we now obtain

$$
\left|v_{x, R}-v(x)\right| \leq \frac{C}{\sigma} R^{\sigma} \tau(x), \quad x \in B_{1}\left(x_{0}\right) .
$$

For $x, y \in B_{1}\left(x_{0}\right)$ with $x \neq y$, take $R=|x-y|$. Then with (18) and the triangle inequality we bound

$$
\begin{aligned}
|v(x)-v(y)| & \leq\left|v_{x, 2 R}-v(x)\right|+\left|v_{x, 2 R}-v_{y, 2 R}\right|+\left|v_{y, 2 R}-v(y)\right| \\
& \leq \frac{C R^{\sigma}(\tau(x)+\tau(y))}{\sigma}+\left|v_{x, 2 R}-v_{y, 2 R}\right| .
\end{aligned}
$$

For any $z \in \mathbb{R}^{n}$, we have

$$
\left|v_{x, 2 R}-v_{y, 2 R}\right| \leq\left|v_{x, 2 R}-v(z)\right|+\left|v_{y, 2 R}-v(z)\right| .
$$

Integrating as $z \in B_{2 R}(x) \cap B_{2 R}(y)$ we get

$$
\begin{aligned}
\left|v_{x, 2 R}-v_{y, 2 R}\right| & \leq \frac{1}{\left|B_{2 R}(x) \cap B_{2 R}(y)\right|}\left(\int_{B_{2 R}(x)}\left|v(z)-v_{x, 2 R}\right| d z+\int_{B_{2 R}(y)}\left|v(z)-v_{y, 2 R}\right| d z\right) \\
& \leq \frac{C R^{\lambda}(\tau(x)+\tau(y))}{R^{n}} \\
& \leq C R^{\sigma}(\tau(x)+\tau(y)) .
\end{aligned}
$$


From (16) and (19) we finally infer

$$
\frac{|v(x)-v(y)|}{|x-y|^{\sigma}} \leq \frac{C}{\sigma}(\tau(x)+\tau(y))=o(1) \log \left(\left|x_{0}\right|+1\right) .
$$

Proof of Theorem 1.1. First we prove that (8) holds. From Lemma 3.1 we have

$$
\liminf _{|x| \rightarrow \infty} \frac{v(x)}{\log |x|} \geq-\beta \text {. }
$$

We assume by contradiction that

$$
\lim _{|x| \rightarrow \infty} \frac{v(x)}{\log |x|} \neq-\beta
$$

Then there exists a sequence of points $\left(x_{k}\right)$ in $\mathbb{R}^{n}$ such that $\left|x_{k}\right| \rightarrow \infty$ and

$$
v\left(x_{k}\right) \geq(-\beta+2 \delta) \log \left|x_{k}\right| \quad \text { for some } \delta>0 .
$$

Indeed, for $\left|x_{k}\right|$ large, by Lemma 3.6

$$
v(x)=v\left(x_{k}\right)+o(1) \log \left|x_{k}\right| \geq(-\beta+\delta) \log \left|x_{k}\right| \quad \text { for } x \in B_{1}\left(x_{k}\right) .
$$

Hence

$$
\lim _{k \rightarrow \infty}\left|x_{k}\right|^{\beta-\delta} \int_{B_{1}\left(x_{k}\right)} e^{v(x)} d x \geq \lim _{k \rightarrow \infty}\left|x_{k}\right|^{\beta-\delta} \int_{B_{1}\left(x_{k}\right)} e^{(-\beta+\delta) \log \left|x_{k}\right|} d x=\left|B_{1}\right| .
$$

This contradicts to Lemma 3.5 with $\rho=1, q=1$ and $0<\varepsilon_{1}<\delta$. Thus (8) is proved.

It remains to show that $\Lambda=\Lambda_{1}(1+\alpha)$ if $p$ is constant. In this case we have

$$
u(x)=\frac{1}{\gamma_{n}} \int_{\mathbb{R}^{n}} \log \left(\frac{1+|y|}{|x-y|}\right)|y|^{n \alpha} e^{n u(y)} d y+C .
$$

Then, we are in position to apply the Pohozaev-type identity of Proposition A.2 to conclude that $\Lambda=\Lambda_{1}(1+\alpha)$.

\section{Proof of Theorem 1.2}

When $\Lambda \in\left(0, \Lambda_{1}(1+\alpha)\right)$ we will look for solutions of the form $u(x)=v(x)-|x|^{2}+c$ where $c \in \mathbb{R}$ and $v$ satisfies the integral equation

$$
v(x)=\frac{1}{\gamma_{n}} \int_{\mathbb{R}^{n}} \log \left(\frac{1}{|x-y|}\right)|y|^{n \alpha} e^{-n|y|^{2}} e^{n(v(y)+c)} d y,
$$

so that in particular

$$
(-\Delta)^{\frac{n}{2}} v(x)=|x|^{n \alpha} e^{-n|x|^{2}} e^{n(v(x)+c)}
$$


Our approach will be based on Schauder's fixed-point theorem (see [9, Theorem 11.3]), an idea already exploited in several works. More precisely we set

$$
X:=\left\{v \in C_{\text {rad }}^{0}\left(\mathbb{R}^{n}\right):\|v\|_{X}<\infty\right\}, \quad\|v\|_{X}:=\sup _{x \in \mathbb{R}^{n}} \frac{|v(x)|}{1+|x|} .
$$

For $v \in X$ we set $c_{v} \in \mathbb{R}$ such that

$$
\int_{\mathbb{R}^{n}}|y|^{n \alpha} e^{-n|y|^{2}} e^{n\left(v(y)+c_{v}\right)} d y=\Lambda,
$$

and define $T=T_{\Lambda}: X \rightarrow X, T v=\bar{v}$ where

$$
\bar{v}(x):=\frac{1}{\gamma_{n}} \int_{\mathbb{R}^{n}} \log \left(\frac{1}{|x-y|}\right)|y|^{n \alpha} e^{-n|y|^{2}} e^{n\left(v(y)+c_{v}\right)} d y .
$$

Lemma 4.1. The operator $T: X \rightarrow X$ is compact.

Proof. Continuity follows by dominated convergence. Let now $\left(v_{k}\right) \subset X$ be a bounded sequence. From the definition of $c_{v_{k}}$ it follows easily that $\left|c_{v_{k}}\right| \leq C$. Therefore,

$$
\left|\bar{v}_{k}(x)\right| \leq C \int_{\mathbb{R}^{n}}|\log | x-y|||y|^{n \alpha} e^{-|y|^{2}} d y \leq C \log (2+|x|) .
$$

Moreover,

$$
\left|\bar{v}_{k}(x)-\bar{v}_{k}(z)\right| \leq C \int_{\mathbb{R}^{n}}\left|\log \left(\frac{|z-y|}{|x-y|}\right)\right||y|^{n \alpha} e^{-|y|^{2}} d y \rightarrow 0, \quad \text { as }|x-z| \rightarrow 0, x, z \in \mathbb{R}^{n} .
$$

Thus, the sequence $\left(\bar{v}_{k}\right)$ is equicontinuous on $\mathbb{R}^{n}$. Hence, by the theorem of Ascoli-Arzelà, up to a subsequence, $\bar{v}_{k} \rightarrow v$ in $C_{\text {loc }}^{0}\left(\mathbb{R}^{n}\right)$ for some $v \in C^{0}\left(\mathbb{R}^{n}\right)$. In particular, $\bar{v}_{k} \rightarrow v$ in $X$, thanks to $(21)$.

Lemma 4.2. The function $\bar{v}$ is radially decreasing.

Proof. Consider the functions

$$
\bar{v}_{\varepsilon}(x):=\frac{1}{\gamma_{n}} \int_{\mathbb{R}^{n} \backslash B_{\varepsilon}} \log \left(\frac{1}{|x-y|}\right)|y|^{n \alpha} e^{-n|y|^{2}} e^{n\left(v(y)+c_{v}\right)} d y .
$$

Differentiating under the integral sign one gets $\Delta \bar{v}_{\varepsilon}<0$, which implies that $\bar{v}_{\varepsilon}$ is radially decreasing. Letting now $\varepsilon \rightarrow 0$ we get $\bar{v}_{\varepsilon} \rightarrow \bar{v}$ by dominated convergence, hence $\bar{v}$ is radially decreasing.

Lemma 4.3. There exist constants $C, C(s)>0$, for $s>0$, such that

$$
\int_{B_{R}}|\Delta \bar{v}| d x \leq C R^{n-2}, \quad \int_{B_{R}^{c}} \frac{|\Delta \bar{v}|}{|x|^{n+s}} d x \leq \frac{C(s)}{R^{s+2}},
$$

for any $v \in X$ and $R>0$. 
Proof. For any $y \in \mathbb{R}^{n}$ we have

$$
\int_{B_{R}} \frac{1}{|x-y|^{2}} d x \leq \int_{B_{R}(y)} \frac{1}{|x-y|^{2}} d x+\int_{B_{R} \cap B_{R}(y)^{c}} \frac{1}{|x-y|^{2}} d x \leq C R^{n-2} .
$$

Then writing with Fubini-Tonelli's theorem

$$
\begin{aligned}
\int_{B_{R}}|\Delta \bar{v}| d x & \leq C \int_{\mathbb{R}^{n}}|y|^{n \alpha} e^{-n|y|^{2}} e^{n\left(v(y)+c_{v}\right)}\left(\int_{B_{R}} \frac{1}{|x-y|^{2}} d x\right) d y \\
& \leq C R^{n-2} \Lambda
\end{aligned}
$$

the first estimate in (22) follows. The second one is proven in the same way since

$$
\int_{B_{R}^{c}} \frac{1}{|x-y|^{2}|x|^{n+s}} d x \leq \int_{B_{R}(y)} \frac{1}{|x-y|^{2} R^{n+s}} d x+\int_{B_{R}^{c} \cap B_{R}(y)^{c}} \frac{1}{R^{2}|x|^{n+s}} d x \leq \frac{C}{R^{s+2}} .
$$

We are now in a position to prove the main a priori estimate.

Proposition 4.1. Assume that $\Lambda \in\left(0, \Lambda_{1}(1+\alpha)\right)$. Then there exists $C>0$ such that for every $(v, t) \in X \times[0,1]$ satisfying $v=t T(v)$ we have $\|v\| \leq C$.

Proof. Assume for the sake of contradiction that there exists $\left(v_{k}, t_{k}\right) \in X \times[0,1]$ such that

$$
v_{k}=t_{k} T\left(v_{k}\right) \text { and }\left\|v_{k}\right\| \rightarrow \infty .
$$

Then $v_{k}$ satisfies the integral equation

$$
v_{k}(x):=\frac{t_{k}}{\gamma_{n}} \int_{\mathbb{R}^{n}} \log \left(\frac{1}{|x-y|}\right)|y|^{n \alpha} e^{-n|y|^{2}} e^{n\left(v_{k}(y)+c_{v_{k}}\right)} d y .
$$

We observe that if

$$
w_{k}:=v_{k}+c_{v_{k}}+\frac{1}{n} \log t_{k} \leq C \quad \text { on } \mathbb{R}^{n},
$$

then from $(23)$

$$
\left|v_{k}(x)\right| \leq \frac{e^{n C}}{\gamma_{n}} \int_{\mathbb{R}^{n}}|\log | x-y|||y|^{n \alpha} e^{-n|y|^{2}} d y \leq C \log (2+|x|),
$$

a contradiction to our assumption $\left\|v_{k}\right\| \rightarrow \infty$. Thus, together with Lemma 4.2 , we have

$$
\max _{\mathbb{R}^{n}} w_{k}=w_{k}(0) \rightarrow \infty
$$

We set

$$
\eta_{k}(x)=w_{k}\left(r_{k} x\right)-w_{k}(0), \quad r_{k}^{1+\alpha}:=e^{-w_{k}(0)} \rightarrow 0 .
$$

Then, on compact sets we have

$$
(-\Delta)^{\frac{n}{2}} \eta_{k}(x)=|x|^{n \alpha} e^{-n\left|r_{k} x\right|^{2}} e^{n \eta_{k}(x)}=(1+o(1))|x|^{n \alpha} e^{n \eta_{k}(x)} .
$$


Moreover by Lemma 4.3 we have

$$
\int_{B_{R}}\left|\Delta \eta_{k}(x)\right| d x \leq C R^{n-2}, \quad \int_{\mathbb{R}^{n} \backslash B_{R}} \frac{\left|\Delta \eta_{k}(x)\right|}{|x|^{2 n-2}} d x \leq \frac{C}{R^{n}} .
$$

Then by elliptic estimates, Proposition A.3 in the appendix, up to a subsequence, $\eta_{k} \rightarrow \eta$ locally uniformly $\mathbb{R}^{n}$ and in $C_{\text {loc }}^{n-1}\left(\mathbb{R}^{n} \backslash\{0\}\right)$. Note further that we have

$$
\begin{aligned}
\int_{\mathbb{R}^{n}}|x|^{n \alpha} e^{n \eta(x)} d x & =\lim _{R \rightarrow \infty} \lim _{k \rightarrow \infty} \int_{B_{R}}|x|^{n \alpha} e^{n \eta_{k}(x)} d x \\
& =\lim _{R \rightarrow \infty} \lim _{k \rightarrow \infty} t_{k} \int_{B_{R r_{k}}}|x|^{n \alpha} e^{-n|x|^{2}} e^{n\left(v_{k}(x)+c_{v_{k}}\right)} d x \\
& \leq \Lambda t_{\infty} .
\end{aligned}
$$

where, up to a subsequence, $t_{\infty}:=\lim _{k \rightarrow \infty} t_{k}$. Thus $t_{\infty} \neq 0$ and

$$
\int_{\mathbb{R}^{n}}|x|^{n \alpha} e^{n \eta} d x<\infty
$$

We claim that $\eta$ is a normal solution to $(-\Delta)^{\frac{n}{2}} \eta=|x|^{n \alpha} e^{n \eta}$, namely

$$
\eta(x)=\frac{1}{\gamma_{n}} \int_{\mathbb{R}^{n}} \log \left(\frac{1+|y|}{|x-y|}\right)|y|^{n \alpha} e^{n \eta(y)} d y+c,
$$

for some $c \in \mathbb{R}$. Since $n \geq 3$ we have that $\Delta \eta_{k} \rightarrow \Delta \eta$ locally uniformly in $\mathbb{R}^{n} \backslash\{0\}$ and by the first estimate in (24) we conclude that $\Delta \eta_{k} \rightarrow \Delta \eta$ in $L_{\text {loc }}^{1}\left(\mathbb{R}^{n}\right)$. Then, also using the second estimate in (24) and recalling that for $\varphi \in \mathcal{S}\left(\mathbb{R}^{n}\right)$ we have

$$
\left|(-\Delta)^{\frac{n-2}{2}} \varphi\right| \leq C(1+|x|)^{-2 n+2}
$$

we get

$$
\begin{aligned}
\int_{\mathbb{R}^{n}}(-\Delta \eta)(-\Delta)^{\frac{n-2}{2}} \varphi d x & =\lim _{k \rightarrow \infty} \int_{\mathbb{R}^{n}}\left(-\Delta \eta_{k}\right)(-\Delta)^{\frac{n-2}{2}} \varphi d x \\
& =\lim _{k \rightarrow \infty} \int_{\mathbb{R}^{n}} \eta_{k}(-\Delta)^{\frac{n}{2}} \varphi d x \\
& =\lim _{k \rightarrow \infty} \int_{\mathbb{R}^{n}}|x|^{n \alpha} e^{-n\left|r_{k} x\right|^{2}} e^{n \eta_{k}} \varphi d x \\
& =\int_{\mathbb{R}^{n}}|x|^{n \alpha} e^{n \eta} \varphi d x
\end{aligned}
$$

In order to prove that (27) implies (26) (compare to Definitions 1.1 and 1.2), we set

$$
\tilde{\eta}(x):=\frac{1}{\gamma_{n}} \int_{\mathbb{R}^{n}} \log \left(\frac{1+|y|}{|x-y|}\right)|y|^{n \alpha} e^{n \eta(y)} d y, \quad p:=\eta-\tilde{\eta} .
$$

Then $p$ satisfies

$$
\int_{B_{R}}|\Delta p| d x \leq C R^{n-2}, \quad \int_{\mathbb{R}^{n}} \frac{|\Delta p|}{1+|x|^{2 n-2}} d x<\infty, \quad \int_{\mathbb{R}^{n}} \Delta p(-\Delta)^{\frac{n-2}{2}} \varphi d x=0,
$$


for every $\varphi \in \mathcal{S}\left(\mathbb{R}^{n}\right)$. Hence $\Delta p \in L_{\frac{n-2}{2}}\left(\mathbb{R}^{n}\right)$ is $\frac{n-2}{2}$-harmonic in $\mathbb{R}^{n}$, which implies that $\Delta p$ is a polynomial (see e.g. the proof of [14, Lemma 2.4]). Now the estimate $\int_{B_{R}}|\Delta p| d x \leq C R^{n-2}$ gives $\Delta p \equiv 0$. Since $\tilde{\eta}(x) \geq-C \log (2+|x|)$ on $\mathbb{R}^{n}$ (see the proof of Lemma 3.1), and $|\cdot|^{n \alpha} e^{n \eta} \in L^{1}\left(\mathbb{R}^{n}\right)$, by Theorem A.1 we get $p \equiv$ const, and (26) follows.

From the Pohozaev identity of Proposition A.2 (case $\mu=0)$ and (25), we infer

$$
\Lambda_{1}(1+\alpha)=\int_{\mathbb{R}^{n}}|x|^{n \alpha} e^{n \eta} d x \leq \Lambda
$$

which contradicts our assumption $\Lambda<\Lambda_{1}(1+\alpha)$.

Proof of Theorem 1.2 (completed). Assume first $\Lambda \in\left(0, \Lambda_{1}(1+\alpha)\right)$. Thanks to Proposition 4.1 we can apply Schauder's theorem, hence $T$ has a fixed point $v$. Then the function $u(x)=$ $v(x)-|x|^{2}+c_{v}$ satisfies (3), as wished.

Now we consider the case $\Lambda=\Lambda_{1}(1+\alpha)$. We fix a sequence $\Lambda_{k} \uparrow \Lambda$, and for each $k$ we apply the previous procedure to find $v_{k}$ fixed point of the corresponding $T_{\Lambda_{k}}$. We claim that

$$
v_{k}(0)+c_{v_{k}} \rightarrow \infty
$$

Otherwise, from (23) we would infer that $v_{k}$ satisfies $\left|v_{k}(x)\right| \leq C \log (2+|x|)$ on $\mathbb{R}^{n}$. Then from the definition of $c_{v_{k}}$ we get $\left|c_{v_{k}}\right| \leq C$. Moreover $\left(v_{k}\right)$ is equicontinuous on $\mathbb{R}^{n}$, and therefore, up to a subsequence, $v_{k} \rightarrow v$ locally uniformly in $\mathbb{R}^{n}$. The limit function $v$ satisfies

$$
v(x)=\frac{1}{\gamma_{n}} \int_{\mathbb{R}^{n}} \log \left(\frac{1}{|x-y|}\right)|y|^{n \alpha} e^{-n|y|^{2}} e^{n(v(y)+c)} d y,
$$

where $c_{v_{k}} \rightarrow c$ and

$$
\int_{\mathbb{R}^{n}}|y|^{n \alpha} e^{-n|y|^{2}} e^{n(v(y)+c)} d y \leq \lim _{k \rightarrow \infty} \Lambda_{k}=\Lambda_{1}(1+\alpha),
$$

a contradiction to Proposition A.2 (case $\mu=n$ ). This proves (28).

Setting

$$
\eta_{k}(x)=v_{k}\left(r_{k} x\right)-v_{k}(0), \quad r_{k}^{1+\alpha}:=e^{-v_{k}(0)-c_{v_{k}}} \rightarrow 0,
$$

as in the proof of Lemma 4.1, one obtains $\eta_{k} \rightarrow \eta$, where $\eta$ is a normal solution to (3).

\section{$5 \quad$ Proof of Theorem 1.3}

We set

$$
X:=\left\{v \in C^{0}\left(\mathbb{R}^{n}\right):\|v\|<\infty\right\}, \quad\|v\|:=\sup _{x \in \mathbb{R}^{n}} \frac{|v(x)|}{1+|x|} .
$$

We fix $\Lambda \in\left(0, \Lambda_{1}\right)$ and $\alpha \in(-1, \infty)$. For $v \in X$ let $c_{v} \in \mathbb{R}$ be determined by

$$
\int_{\mathbb{R}^{n}}|y|^{n \alpha} e^{-n|y|^{2}} e^{n\left(v(y)+c_{v}\right)} d y=\Lambda
$$

and let $v^{*} \in \mathbb{R}$ be given by

$$
v^{*}:=\sup _{|x| \leq 1} e^{\frac{2}{1+\alpha}\left(v(x)+c_{v}\right)} .
$$


We define $T: X \rightarrow X, v \mapsto \bar{v}$ where

$$
\bar{v}(x):=\frac{1}{\gamma_{n}} \int_{\mathbb{R}^{n}} \log \left(\frac{1+|y|}{|x-y|}\right)|y|^{n \alpha} e^{-n|y|^{2}} e^{n\left(v(y)+c_{v}\right)} d y+v^{*} x_{1} .
$$

Notice that

$$
(-\Delta)^{\frac{n}{2}} \bar{v}(x)=|x|^{n \alpha} e^{n\left(v(x)-|x|^{2}+c_{v}\right)} .
$$

We will look for solutions of the form

$$
u(x)=v(x)-|x|^{2}+c_{v} .
$$

Lemma 5.1. The operator $T: X \rightarrow X$ is compact.

Proof. As in Lemma 4.1 continuity follows by dominated convergence. Moreover given a bounded sequence $\left(v_{k}\right) \subset X$, from the definition of $c_{v_{k}}$ it follows that $\left|c_{v_{k}}\right| \leq C$. This in turn implies $\left|v_{k}^{*}\right| \leq C$. If we set $\tilde{v}_{k}(x):=\bar{v}_{k}(x)-v_{k}^{*} x_{1}$, we get $\left|\tilde{v}_{k}(x)\right| \leq C \log (2+|x|)$, and the sequence $\left(\tilde{v}_{k}\right)$ is equicontinuous, with the same proof as in Lemma 4.1. In particular, up to a subsequence, $\tilde{v}_{k} \rightarrow \tilde{v}$ in $X$. Since, up to a subsequence, $\left\|v_{k}^{*} x_{1}-c_{0} x_{1}\right\| \rightarrow 0$ for some $c_{0}>0$, we conclude that $\bar{v}_{k} \rightarrow \tilde{v}+c_{0} x_{1}$ in $X$.

The proof of Theorem 1.3 follows at once from the Schauder fixed-point theorem and the following a priori estimate.

Proposition 5.1. There exists $C>0$ such that

$$
\|v\| \leq C \quad \text { for every }(v, t) \in X \times(0,1] \text { with } v=t T(v) .
$$

The proof of Proposition 5.1 will be based on the following three lemmas.

Lemma 5.2. For every $R>0$ there exists a constant $C(R)>0$ such that for every $(t, v) \in$ $(0,1] \times X$ with $v=t T(v)$, that is

$$
v(x):=\frac{t}{\gamma_{n}} \int_{\mathbb{R}^{n}} \log \left(\frac{1+|y|}{|x-y|}\right)|y|^{n \alpha} e^{-n|y|^{2}} e^{n\left(v(y)+c_{v}\right)} d y+t v^{*} x_{1},
$$

we have

$$
w:=v+c_{v}+\frac{1}{n} \log t \leq C(R) \quad \text { on } B_{R} .
$$

Proof. Assume by contradiction that for a sequence $\left(t_{k}, v_{k}\right) \in(0,1] \times X$ such that $v_{k}=t_{k} T\left(v_{k}\right)$ one has

$$
\max _{\bar{B}_{2 R_{0}}} w_{k}=: w_{k}\left(\xi_{k}\right)=v_{k}\left(\xi_{k}\right)+c_{v_{k}}+\frac{1}{n} \log t_{k} \rightarrow \infty
$$

for some $R_{0}>0$. We set $x_{k}:=\xi_{k}$ and $s_{k}=0$ if $\left|\xi_{k}\right| \not \neg 2 R_{0}$, and otherwise we let $x_{k} \in B_{R_{0}}\left(\xi_{k}\right)$ and $s_{k} \in\left[0, R_{0}\right]$ be such that (see [1])

$$
\left(R_{0}-s_{k}\right) e^{w_{k}\left(x_{k}\right)}=\left(R_{0}-s_{k}\right) \max _{\bar{B}_{s_{k}}\left(\xi_{k}\right)} e^{w_{k}}=\max _{s \in\left[0, R_{0}\right]}\left(\left(R_{0}-s\right) \max _{\bar{B}_{s}\left(\xi_{k}\right)} e^{w_{k}}\right)=: L_{k}
$$


Then $L_{k} \rightarrow \infty$, and

$$
w_{k}\left(x_{k}+\mu_{k} x\right)-w_{k}\left(x_{k}\right) \leq \log 2 \quad \text { for }|x| \leq \frac{L_{k}}{2}, \quad \mu_{k}:=\frac{R_{0}-s_{k}}{L_{k}} .
$$

We distinguish the following cases.

Case 1 Up to a subsequence $\left|x_{k}\right|^{1+\alpha} e^{w_{k}\left(x_{k}\right)} \rightarrow c_{0} \in[0, \infty)$.

In this case we have $x_{k}=\xi_{k} \rightarrow 0$, which implies that $w_{k} \leq w_{k}\left(x_{k}\right)$ on $B_{R_{0}}$. We set

$$
\eta_{k}(x):=w_{k}\left(r_{k} x\right)-w_{k}\left(x_{k}\right), \quad r_{k}^{1+\alpha}:=e^{-w_{k}\left(x_{k}\right)} .
$$

It follows from the definition of $r_{k}$ that $\left|x_{k}\right|=O\left(r_{k}\right)$. Therefore, on any compact set

$$
(-\Delta)^{\frac{n}{2}} \eta_{k}(x)=|x|^{n \alpha} e^{n \eta_{k}(x)} e^{-n\left|r_{k} x\right|^{2}}=(1+o(1))|x|^{n \alpha} e^{n \eta_{k}(x)} .
$$

Since $\alpha>-1$ and $\eta_{k} \leq \log 2$ for large $k$ on any compact set, from (32) we obtain

$$
\left\|(-\Delta)^{\frac{n}{2}} \eta_{k}\right\|_{L^{p}\left(B_{R}\right)} \leq C(p, R) \text { for } 1 \leq p<\bar{p},
$$

and

$$
\left\|(-\Delta)^{\frac{n}{2}} \eta_{k}\right\|_{L^{\infty}(K)} \leq C(K) \text { for every } K \Subset \mathbb{R}^{n} \backslash\{0\} .
$$

Moreover, differentiating in (30) as in Lemma 4.3 (notice that the part $v^{*} x_{1}$ does not play a role in $\Delta \eta_{k}$ ), we obtain

$$
\int_{B_{R}}\left|\Delta \eta_{k}(x)\right| d x \leq C R^{n-2}, \quad \int_{\mathbb{R}^{n} \backslash B_{R}} \frac{\left|\Delta \eta_{k}(x)\right|}{|x|^{2 n-2}} d x \leq \frac{C}{R^{n}} .
$$

We also have $\eta_{k}\left(\bar{x}_{k}\right)=0$, where $\bar{x}_{k}:=\frac{x_{k}}{r_{k}}$ satisfies $\left|\bar{x}_{k}\right|=O(1)$. Then by Proposition A.3, up to a subsequence, $\eta_{k} \rightarrow \eta$ in $C_{\text {loc }}^{0}\left(\mathbb{R}^{n}\right) \cap C_{\text {loc }}^{n-1}\left(\mathbb{R}^{n} \backslash\{0\}\right)$ and $\Delta \eta_{k} \rightarrow \Delta \eta$ in $L_{\text {loc }}^{1}\left(\mathbb{R}^{n}\right)$ for some $\eta$.

Then, with the same argument of Lemma 4.1 we obtain that $\eta$ satisfies the integral equation

$$
\eta(x)=\frac{1}{\gamma_{n}} \int_{\mathbb{R}^{n}} \log \left(\frac{1+|y|}{|x-y|}\right)|y|^{n \alpha} e^{n \eta(y)} d y+c .
$$

In particular, differentiating (34) we obtain that for every $R>0$

$$
\int_{B_{R}}|\nabla \eta(x)| d x \leq C R^{n-1}
$$

Using (30) one obtains for every $R>0$

$$
\begin{aligned}
\int_{B_{R}}\left|\nabla \eta_{k}(x)-t_{k} r_{k} v_{k}^{*} e_{1}\right| d x & \leq t_{k} \int_{B_{R}} \int_{\mathbb{R}^{n}} \frac{r_{k}}{\left|x_{k}+r_{k} x-y\right|}|y|^{n \alpha} e^{-n|y|^{2}} e^{n\left(v_{k}(y)+c_{v_{k}}\right)} d y d x \\
& \leq C R^{n-1} .
\end{aligned}
$$

Therefore, for every $R>0$

$$
\lim _{k \rightarrow \infty} t_{k} r_{k} v_{k}^{*}\left|B_{R}\right| \leq \lim _{k \rightarrow \infty} \int_{B_{R}}\left(\left|\nabla \eta_{k}(x)-t_{k} r_{k} v_{k}^{*} e_{1}\right|+\left|\nabla \eta_{k}(x)\right|\right) d x \leq C R^{n-1}
$$


This shows that

$$
\lim _{k \rightarrow \infty} t_{k} r_{k} v_{k}^{*}=0
$$

Since $t_{k} \rightarrow t_{\infty} \neq 0$, we must have

$$
\lim _{k \rightarrow \infty} r_{k} v_{k}^{*}=0
$$

This is a contradiction since from $\liminf _{k \rightarrow \infty} t_{k}>0$ we infer $v_{k}^{*} \geq \frac{1}{C} e^{\frac{2}{1+\alpha} w_{k}\left(x_{k}\right)}$, hence

$$
r_{k} v_{k}^{*} \geq \frac{1}{C} e^{\frac{1}{1+\alpha} w_{k}\left(x_{k}\right)} \rightarrow \infty .
$$

Case 2 Up to a subsequence $\left|x_{k}\right|^{1+\alpha} e^{w_{k}\left(x_{k}\right)} \rightarrow \infty$.

We set

$$
\eta_{k}(x)=w_{k}\left(x_{k}+r_{k} x\right)-w_{k}\left(x_{k}\right)
$$

where

$$
r_{k}:=\left|x_{k}\right|^{-\alpha} e^{-w_{k}\left(x_{k}\right)} .
$$

Notice that by (31) for every $R>0$ we have $\eta_{k}(x) \leq \log 2$ on $B_{R}$ for $k \geq k_{0}(R)$. Moreover $r_{k}=o\left(\left|x_{k}\right|\right)$ and we compute

$$
(-\Delta)^{\frac{n}{2}} \eta_{k}(x)=e^{-n\left|x_{0}\right|^{2}}(1+o(1))\left|\frac{x_{k}}{\left|x_{k}\right|}+\frac{r_{k}}{\left|x_{k}\right|} x\right|^{n \alpha} e^{n \eta_{k}}=\left(c_{0}+o(1)\right) e^{n \eta_{k}}
$$

on compact sets, where $x_{k} \rightarrow x_{0}$ and $c_{0}:=e^{-n\left|x_{0}\right|^{2}}$. Then, similar to Case 1 , we obtain $\eta_{k} \rightarrow \eta$ where $\eta$ is a normal solution to

$$
(-\Delta)^{\frac{n}{2}} \eta=c_{0} e^{n \eta} \quad \text { in } \mathbb{R}^{n}, \quad \int_{\mathbb{R}^{n}} e^{n \eta} d x<\infty,
$$

that is, $\eta$ is a spherical, a contradiction as $\Lambda<\Lambda_{1}$.

Lemma 5.3. There exists $C>0$ such that for every $(t, v) \in(0,1] \times X$ such that $v=t T(v)$ one has $v^{*} \leq C$ and $c_{v} \leq C$.

Proof. Take $(t, v) \in(0,1] \times X$ be such that $v=t T(v)$ and let $w$ be as in Lemma 5.2. For $|x| \leq 1$ we obtain from $(30)$

$$
\begin{aligned}
v(x)-t v^{*} x_{1} & =\frac{1}{\gamma_{n}}\left(\int_{|y|<2}+\int_{|y|>2}\right) \log \left(\frac{1+|y|}{|x-y|}\right)|y|^{n \alpha} e^{-n|y|^{2}} e^{n w(y)} d y \\
& =O(1) \int_{|y|<2} \log \left(\frac{1+|y|}{|x-y|}\right)|y|^{n \alpha} d y+O(1) \int_{|y|>2}|y|^{n \alpha} e^{-n|y|^{2}} e^{n w(y)} d y \\
& =O(1),
\end{aligned}
$$

where the last equality follows from (29), while in the second inequality we have used that $w \leq C$ on $B_{2}$ thanks to Lemma 5.2 , and the estimate $\log \left(\frac{1+|y|}{|x-y|}\right)=O(1)$ for $(x, y) \in B_{1} \times B_{2}^{c}$. Therefore,

$$
v(x)+c_{v}=c_{v}+t v^{*} x_{1}+O(1) \quad \text { on } B_{1} .
$$

This and (29) imply that $v+c_{v} \leq C$ on $B_{1}$, which is equivalent to $v^{*} \leq C$. In particular, from (35), we have $v=O(1)$ in $B_{1}$, and (29) yields $c_{v} \leq C$. 
Lemma 5.4. For every $\varepsilon>0$ there exists $R>0$ such that the following holds: Given $(t, v) \in$ $(0,1] \times X$ with $v=t T(v)$ one has

$$
\int_{|x|>R} e^{-n|x|^{2}}|x|^{n \alpha} e^{n w(x)} d x<\varepsilon
$$

where $w:=v+c_{v}+\frac{1}{n} \log t$.

Proof. We use a Pohozaev type identity for the integral equation

$$
\tilde{w}(x)=\frac{1}{\gamma_{n}} \int_{\mathbb{R}^{n}} \log \left(\frac{1}{|x-y|}\right) e^{-n|y|^{2}-t v^{*} y_{1}}|y|^{n \alpha} e^{n \tilde{w}(y)} d y+n c_{v}+\frac{1}{n} \log t,
$$

where $\tilde{w}:=w+t v^{*} x_{1}$. Since $(t, v) \in(0,1] \times X$, we can apply Proposition A.1 to get

$$
\begin{aligned}
t \Lambda\left(t \Lambda-\Lambda_{1}\right) & =c \int_{\mathbb{R}^{n}} y \cdot \nabla\left(e^{-n|y|^{2}-t v^{*} y}|y|^{n \alpha}\right) e^{n \tilde{w}(y)} d y \\
& =c n \alpha \int_{\mathbb{R}^{n}}|y|^{n \alpha} e^{-n|y|^{2}} e^{n w(y)} d y-c \int_{\mathbb{R}^{n}}\left(2 n|y|^{2}+t v^{*} y\right)_{1} e^{-n|y|^{2}}|y|^{n \alpha} e^{n w(y)} d y \\
& =\operatorname{cn\alpha t} \Lambda-c\left(\int_{B_{R_{0}}}+\int_{B_{R_{0}}^{c}}\right)\left(2 n|y|^{2}+t v^{*} y_{1}\right) e^{-n|y|^{2}}|y|^{n \alpha} e^{n w(y)} d y \\
& =\text { c } \operatorname{cn} \alpha t \Lambda-c\left(I_{1}+I_{2}\right),
\end{aligned}
$$

where $R_{0}>0$ is such that $2 n|y|^{2}+t v^{*} y_{1} \geq|y|^{2}$ on $B_{R_{0}}^{c}$. We observe that $\left|I_{1}\right| \leq C\left(R_{0}, \Lambda, n\right)$, thanks to Lemma 5.2 and the estimate $v^{*} \leq C$ of Lemma 5.3. Therefore, for $R>R_{0}$ we obtain

$$
\begin{aligned}
\int_{B_{R}^{c}} e^{-n|y|^{2}}|y|^{n \alpha} e^{n w(y)} d y & \leq \frac{1}{R^{2}} \int_{B_{R}^{c}}|y|^{2} e^{-n|y|^{2}}|y|^{n \alpha} e^{n w(y)} d y \\
& \leq \frac{1}{R^{2}} \int_{B_{R}^{c}}\left(2 n|y|^{2}+t v^{*} y_{1}\right) e^{-n|y|^{2}}|y|^{n \alpha} e^{n w(y)} d y \\
& \leq \frac{1}{R^{2}} \int_{B_{R_{0}}^{c}}\left(2 n|y|^{2}+t v^{*} y_{1}\right) e^{-n|y|^{2}}|y|^{n \alpha} e^{n w(y)} d y \\
& \leq \frac{1}{R^{2}} C\left(R_{0}, \Lambda, n\right) .
\end{aligned}
$$

This proves the lemma.

Proof of Proposition 5.1. Since $v^{*} \leq C$, thanks to Lemma 5.3, it is sufficient to show that $\tilde{v}:=v-t v^{*} x_{1}$ is bounded in $X$ (we want to show that $|\tilde{v}(x)| \leq C \log (2+|x|)$ ). We have

$$
\tilde{v}(x):=\int_{\mathbb{R}^{n}} \log \left(\frac{1+|y|}{|x-y|}\right) K(y) e^{n \tilde{v}(y)} d y, \quad K(y):=\frac{t_{k}}{\gamma_{n}}|y|^{n \alpha} e^{-n|y|^{2}} e^{n c_{v}+n t v^{*} y_{1}} .
$$

As $v^{*} \leq C$ and $c_{v} \leq C$, there exists $R>0$ such that

$$
K(y) \leq e^{-2|y|^{2}} \quad \text { on } B_{R}^{c} .
$$


By Lemma 5.4 we can also assume that

$$
\int_{B_{R}^{c}} K e^{n \tilde{v}} d y \leq \frac{1}{4}
$$

Then, as in Lemma 3.4 one obtains

$$
\tilde{v}(x) \leq\left(-t \Lambda+\frac{1}{4}\right) \log |x|+\int_{B_{1}(x)} \log \left(\frac{1}{|x-y|}\right) K(y) e^{n \tilde{v}(y)} d y, \quad|x| \geq R .
$$

In the spirit of Lemma 3.5 we get

$$
\int_{B_{1}\left(x_{0}\right)} e^{2 n \tilde{v}(z)} d z \leq C\left|x_{0}\right|^{\frac{1}{4}}, \quad\left|x_{0}\right| \geq R+2 .
$$

By (37) and Hölder inequality, from (38)

$$
\tilde{v}(x) \leq \frac{1}{4} \log |x|+C, \quad|x| \geq R+2 .
$$

Therefore,

$$
|\tilde{v}(x)| \leq C \int_{\mathbb{R}^{n}}\left|\log \left(\frac{1+|y|}{|x-y|}\right)\right| e^{-|y|^{2}} d y \leq C \log (2+|x|),
$$

thanks to Lemma 5.2.

\section{A Appendix}

\section{A.1 A Pohozaev-type result}

Proposition A.1. Assume that $K \in W_{\text {loc }}^{1,1}\left(\mathbb{R}^{n} \backslash\{0\}\right) \cap L_{\text {loc }}^{p}\left(\mathbb{R}^{n}\right)$ for some $p>1, n \geq 2$. Let $\eta$ be a solution to the integral equation

$$
\eta(x)=\frac{1}{\gamma_{n}} \int_{\mathbb{R}^{n}} \log \left(\frac{1+|y|}{|x-y|}\right) K(y) e^{n \eta(y)} d y+c
$$

for some $c \in \mathbb{R}$, with $K e^{n \eta} \in L^{1}\left(\mathbb{R}^{n}\right)$ and $(\nabla K \cdot x) e^{n \eta} \in L^{1}\left(\mathbb{R}^{n}\right)$. If there exists $R_{0}, \varepsilon>0$ such that

$$
|K(x)| e^{n \eta(x)} \leq \frac{1}{|x|^{n+\varepsilon}} \quad \text { for }|x| \geq R_{0},
$$

then, denoting $\Lambda:=\int_{\mathbb{R}^{n}} K(x) e^{n \eta(x)} d x$, we have

$$
\frac{\Lambda}{\gamma_{n}}\left(\Lambda-2 \gamma_{n}\right)=\frac{2}{n} \int_{\mathbb{R}^{n}}(x \cdot \nabla K(x)) e^{n \eta(x)} d x,
$$

Proof. Noticing that $\eta \in C^{0}\left(\mathbb{R}^{n}\right) \cap W_{\text {loc }}^{1,1}\left(\mathbb{R}^{n}\right)$, in the spirit of $[24$, Theorem 1.1], for any $R>0$ we can multiply $\nabla \eta$ by $x \cdot \nabla \eta$ and integrate on $B_{R}$ using the divergence theorem to get

$$
\begin{aligned}
& \int_{B_{R}} K e^{n \eta} d x+\frac{1}{n} \int_{B_{R}}(x \cdot \nabla K(x)) e^{n \eta} d x-\frac{R}{n} \int_{\partial B_{R}} K e^{n \eta} d \sigma(x) \\
& =\frac{1}{2 \gamma_{n}} \int_{B_{R}} \int_{B_{R}^{c}} \frac{(x-y) \cdot(x+y)}{|x-y|^{2}} K(y) e^{n \eta(y)} K(x) e^{n \eta(x)} d y d x \\
& \quad+\frac{1}{2 \gamma_{n}} \int_{\mathbb{R}^{n}} K(y) e^{n \eta(y)} d y \int_{B_{R}} K(x) e^{n \eta(x)} d x .
\end{aligned}
$$


It follows from (40) that the boundary term and the double integral term go to 0 as $R \rightarrow \infty$. Therefore, taking $R \rightarrow \infty$, we obtain (41).

We are interested in the following special cases of the above proposition.

Proposition A.2. Given $n \geq 2, \alpha>-1, \mu \geq 0$ let $\eta$ be a solution to (39) with $c \in \mathbb{R}$, $K:=|\cdot|^{n \alpha} e^{-\mu|\cdot|^{2}}$ and

$$
\Lambda:=\int_{\mathbb{R}^{n}} K(x) e^{n \eta(x)} d x<+\infty .
$$

Then, $\Lambda \leq \Lambda_{1}(1+\alpha)$ and the equality holds if and only if $\mu=0$.

Proof. First, we claim that (40) holds. If $\mu=0$, then $\eta$ is a normal solution to $(-\Delta)^{\frac{n}{2}} \eta=$ $|x|^{n \alpha} e^{n \eta}$ and the classification part of Theorem 1.1 implies that

$$
\lim _{|x| \rightarrow \infty} \frac{\eta(x)}{\log |x|}=-\beta, \quad \beta:=\frac{\Lambda}{\gamma_{n}} .
$$

Moreover, arguing as in Lemma 3.1 we get

$$
v(x) \geq-\beta \log |x|+c, \quad|x| \geq 1,
$$

and from $K e^{n \eta} \in L^{1}$ we find that $\beta>1+\alpha$. Then (40) follows at once form (42). If $\mu>0$, we get (42) from Remark 3.2 and the function $K e^{n \eta}$ decays exponentially at infinity, so that (40) trivially holds. Observe now that the condition $(\nabla K \cdot x) e^{n \eta} \in L^{1}\left(\mathbb{R}^{n}\right)$ is satisfied, since $\nabla K \cdot x=n \alpha K$ for $\mu=0$, and since $(\nabla K \cdot x) e^{n \eta}$ decays exponentially for $\mu>0$ thanks to (42). Then, we can apply Proposition A.1 to get

$$
\begin{aligned}
\frac{\Lambda}{\gamma_{n}}\left(\Lambda-2 \gamma_{n}\right) & =\frac{2}{n} \int_{\mathbb{R}^{n}}\left(x \cdot \nabla\left(|x|^{n \alpha} e^{-\mu|x|^{2}}\right)\right) e^{n \eta(x)} d x \\
& =2 \alpha \Lambda-\frac{4 \mu}{n} \int_{\mathbb{R}^{n}}|x|^{n \alpha+2} e^{-\mu|x|^{2}} e^{n \eta(x)} d x \\
& \leq 2 \alpha \Lambda,
\end{aligned}
$$

with equality holding iff $\mu=0$. Since $\Lambda_{1}=2 \gamma_{n}$, the proof is complete.

\section{A.2 A Liouville-type theorem}

Lemma A.1. Let $\alpha \in(-1,0)$ and $u$ be a measurable function such that $|\cdot|{ }^{n \alpha} e^{n u} \in L^{1}\left(\mathbb{R}^{n}\right)$. Then for any $x \in \mathbb{R}^{n}$ we have

$$
f_{B_{R}(x)} u^{+} d y \rightarrow 0
$$

as $R \rightarrow \infty$.

Proof. Fix $x \in \mathbb{R}^{n}$. With $n u^{+} \leq e^{n u}$, multiplying and dividing by $|y|^{n \alpha}$ we get

$$
\begin{aligned}
n f_{B_{R}(x)} u^{+} d y & \leq f_{B_{R}(x)} e^{n u} d y \\
& \leq \frac{C(R+|x|)^{-n \alpha}}{R^{n}} \int_{B_{R}(x)}|y|^{n \alpha} e^{n u} d y \\
& \leq \frac{C(R+|x|)^{-n \alpha}}{R^{n}},
\end{aligned}
$$


where we used that for $y \in B_{R}(x)$ we have $|y| \leq R+|x|$ and that $\int_{\mathbb{R}^{n}}|x|^{n \alpha} e^{n u} d x<\infty$. The claim follows letting $R \rightarrow \infty$ since $\alpha \in(-1,0)$.

Theorem A.1. Let $\alpha>-1, m \geq 1$ and consider $h: \mathbb{R}^{n} \rightarrow \mathbb{R}$ with $(-\Delta)^{\frac{m}{2}} h=0$ in the sense of Definition 1.1. If $m$ is even, assume further that $h(x) \leq u+C \log (1+|x|)+C$ for any $x \in \mathbb{R}^{n}$, with $\int_{\mathbb{R}^{n}}|x|^{n \alpha} e^{n u} d x<\infty$. Then $h$ is a polynomial of degree at most $m-1$.

Proof. If $m \geq 1$ is even, the proof is almost identical to the one of Theorem 6 in [18], the only difference being the estimate of the term $f_{B_{R}(x)} u^{+} d y \rightarrow 0$ for $\alpha \in(-1,0)$, which is true thanks to Lemma A.1. In the case $m \geq 1$ odd, notice that $h \in L_{\frac{m}{2}}\left(\mathbb{R}^{n}\right)$ by Definition 1.1. This implies that $h$ is a polynomial of degree at most $m-1$ (see e.g. the proof of [14, Lemma 2.4]).

\section{A.3 A non-local elliptic estimate}

Proposition A.3. Assume $n \geq 3$. Let $\left(u_{k}\right) \subset L_{\frac{n}{2}}\left(\mathbb{R}^{n}\right)$ be a sequence of solutions to

$$
(-\Delta)^{\frac{n}{2}} u_{k}=f_{k} \quad \text { in } \mathbb{R}^{n}
$$

for some $f_{k} \in L^{1}\left(\mathbb{R}^{n}\right)$ satisfying

$$
\left\|f_{k}\right\|_{L^{1}\left(\mathbb{R}^{n}\right)} \leq C, \quad\left\|f_{k}\right\|_{L^{p}\left(B_{R}\right)} \leq C, \quad\left\|f_{k}\right\|_{L^{\infty}(A)} \leq C,
$$

for some $p>1, R>0$ and an open set $A \Subset \mathbb{R}^{n} \backslash\{0\}$. Assume further that

$$
u_{k} \leq u_{k}(0)=0 \text { in } B_{R} \text { and } \int_{B_{R}}\left|\Delta u_{k}\right| d x \leq C .
$$

Then the sequence $\left(u_{k}\right)$ is bounded $C_{\mathrm{loc}}^{0, \sigma_{1}}\left(B_{R}\right)$ and in $C_{\mathrm{loc}}^{n-1, \sigma_{2}}(A)$ for some $\sigma_{1}=\sigma_{1}(p) \in(0,1)$ and for every $\sigma_{2} \in(0,1)$.

Proof. We set

$$
v_{k}(x):=\frac{1}{\gamma_{n}} \int_{\mathbb{R}^{n}} \log \left(\frac{1+|y|}{|x-y|}\right) f_{k}(y) d y, \quad p_{k}:=u_{k}-v_{k} .
$$

Then by [14, Lemma 2.4] we have that $p_{k}$ is a polynomial of degree at most $n-1$. It follows from the assumptions on $f_{k}$ and from (45) that $\left(v_{k}\right)$ is bounded in $C_{\mathrm{loc}}^{0, \sigma_{1}}\left(B_{R}\right)$ and in $C_{\mathrm{loc}}^{n-1, \sigma_{2}}(A)$. Therefore, $p_{k}$ satisfies

$$
\sup _{B_{R}} p_{k} \leq C(R), \quad\left|p_{k}(0)\right| \leq C, \quad \int_{B_{\frac{R}{2}}}\left|\Delta p_{k}\right| d x \leq C .
$$

Hence, $\left(p_{k}\right)$ is bounded in $C_{\text {loc }}^{\ell}\left(\mathbb{R}^{n}\right)$ for every $\ell \in \mathbb{N}$.

\section{A.4 Some integral estimates}

Lemma A.2. There exists $C>0$ such that for every $\rho \in(0,1]$ we have for any $x, \xi \in \mathbb{R}^{n}$

$$
\int_{B_{\rho}(x)} \int_{B_{\rho}(x)}\left|\log \left(\frac{|z-\xi|}{|y-\xi|}\right)\right| d z d y \leq C \rho^{2 n}
$$


ii) If $|x-\xi| \geq 2 \sqrt{\rho}$ then

$$
\int_{B_{\rho}(x)} \int_{B_{\rho}(x)}\left|\log \left(\frac{|z-\xi|}{|y-\xi|}\right)\right| d z d y \leq C \rho^{2 n+\frac{1}{2}} .
$$

iii)

$$
\int_{B_{\rho}(x)} \int_{B_{\rho}(x)}\left|\log \left(\frac{|z-\xi|}{|y-\xi|}\right)\right| d z d y=o(1) \rho^{2 n}, \quad o(1) \stackrel{|x-\xi| \rightarrow \infty}{\longrightarrow} 0 .
$$

Proof. Under the rescaling

$$
y^{\prime}=\frac{y-x}{\rho} \quad z^{\prime}=\frac{z-x}{\rho} \quad \xi^{\prime}:=\frac{\xi-x}{\rho},
$$

i) will be equivalent to showing

$$
\int_{B_{1}(0)} \int_{B_{1}(0)}\left|\log \left(\frac{\left|z^{\prime}-\xi^{\prime}\right|}{\left|y^{\prime}-\xi^{\prime}\right|}\right)\right| d z^{\prime} d y^{\prime} \leq C,
$$

independently on $x, \xi^{\prime}$.

If $\left|\xi^{\prime}\right| \geq 2$, then we suffice to apply the triangular inequality to get $\left|\log \left(\frac{\left|z^{\prime}-\xi^{\prime}\right|}{\mid y^{\prime}-\xi^{\prime}} \mid\right)\right| \leq \log 3$.

On the other hand, for $\left|\xi^{\prime}\right| \leq 2$ one has

$$
\begin{aligned}
\int_{B_{1}(0)} \int_{B_{1}(0)}\left|\log \left(\frac{\left|z^{\prime}-\xi^{\prime}\right|}{\left|y^{\prime}-\xi^{\prime}\right|}\right)\right| d z^{\prime} d y^{\prime} & \leq \int_{B_{3}\left(\xi^{\prime}\right)} \int_{B_{3}\left(\xi^{\prime}\right)}\left|\log \left(\frac{\left|z^{\prime}-\xi^{\prime}\right|}{\left|y^{\prime}-\xi^{\prime}\right|}\right)\right| d z^{\prime} d y^{\prime} \\
& =\int_{B_{3}(0)} \int_{B_{3}(0)}\left|\log \left(\frac{\left|z^{\prime \prime}\right|}{\left|y^{\prime \prime}\right|}\right)\right| d z^{\prime \prime} d y^{\prime \prime} \\
& \leq 2\left|B_{3}(0)\right| \int_{B_{3}(0)}|\log | z^{\prime \prime}|| d z^{\prime \prime \prime} \\
& \leq C .
\end{aligned}
$$

To prove $i$ i) first notice that for $|x-\xi| \geq 2 \sqrt{\rho}$ one has

$$
\frac{|z-\xi|}{|y-\xi|} \leq \frac{|y-\xi|+|z-y|}{|y-\xi|} \leq 1+2 \sqrt{\rho} .
$$

Exchanging the role of $y$ and $z$ we find the same estimate for $\frac{|y-\xi|}{|z-\xi|}$. Therefore

$$
\left|\log \left(\frac{|z-\xi|}{|y-\xi|}\right)\right| \leq \log (1+2 \sqrt{\rho}) \leq 2 \sqrt{\rho} .
$$

The proof follows immediately.

As for the proof of $i i i)$ it suffices to notice that as $|x-\xi| \rightarrow \infty$ we have

$$
\frac{|z-\xi|}{|y-\xi|} \rightarrow 1 \quad \text { uniformly for } y, z \in B_{\rho}(x) \subset B_{1}(x) \text {. }
$$




\section{References}

[1] Adimurthi, F. Robert, M. Struwe, Concentration phenomena for Liouville's equation in dimension 4, J. Eur. Math. Soc. 8, (2006), 171-180.

[2] D. Bartolucci, On the best pinching constant of conformal metrics on $S^{2}$ with one and two conical singularities, J. Geom. Anal. 23 (2013), no. 2, 855-877.

[3] T. Branson, Sharp inequality, the functional determinant and the complementary series, Trans. Amer. Math. Soc. 347 (1995), 3671-3742.

[4] T. Branson, B. Oersted, Explicit functional determinants in four dimensions, Comm. Partial Differential Equations 16 (1991), 1223-1253.

[5] S.-Y. A. Chang, W. Chen, A note on a class of higher order conformally covariant equations, Discrete Contin. Dynam. Systems 7 (2001), no. 2, 275-281.

[6] W. Chen, C. LI, Qualitative properties of solutions to some nonlinear elliptic equations in $\mathbb{R}^{2}$, Duke Math. J. 71 (1993), 427-439.

[7] C. Fefferman, K. Hirachi, Ambient metric construction of Q-curvature in conformal and CR geomotries, Mathematical Research Letters 10 (2003), 819-831.

[8] M. Giaquinta, L. Martinazzi, An introduction to the regularity theory for elliptic systems, harmonic maps and minimal graphs, Second edition, [Lecture Notes. Scuola Normale Superiore di Pisa (New Series)], 11. Edizioni della Normale, Pisa, 2012. xiv+366 pp. ISBN: 978-88-7642-442-7; 97888-7642-443-4 35-02.

[9] D. Gilbarg, N. Trudinger, Elliptic partial differential equations of second order, Reprint of the 1998 edition. Classics in Mathematics, Springer-Verlag, Berlin, 2001. xiv+517 pp. ISBN: 3540-41160-7.

[10] E. A. Gorin, Asymptotic properties of polynomials and algebraic functions of several variables, Russ. Math. Surv. 16 (1), 93-119 (1961).

[11] X. Huang, D. Ye, Conformal metrics in $\mathbb{R}^{n}$ with constant $Q$-curvature and arbitrary volume, Calc. Var. Partial Differential Equations 54 (2015), 3373-3384.

[12] A. Hyder, Existence of entire solutions to a fractional Liouville equation in $\mathbb{R}^{n}$, Rend. Lincei. Mat. Appl. 27 (2016), 1-14.

[13] A. HydER, Conformally Euclidean metrics on $\mathbb{R}^{n}$ with arbitrary total Q-curvature, Analysis \& PDE. 10 (2017), no. 3, 635-652.

[14] A. Hyder, Structure of conformal metrics on $\mathbb{R}^{n}$ with constant Q-curvature, to appear in Differential and Integral Equations (2019), arXiv: 1504.07095 (2015).

[15] A. Hyder, L. Martinazzi, Conformal metrics on $\mathbb{R}^{n}$ with constant Q-curvature, prescribed volume, asymptotic behavior, Discr. Cont. Dynamical Systems - A 35 (2015), 283-299.

[16] T. Jin, A. Maalaoui, L. Martinazzi, J. Xiong, Existence and asymptotics for solutions of a non-local Q-curvature equation in dimension three, Calc. Var. Partial Differential Equations 52 (2015) no. 3-4, 469-488.

[17] C. S. Lin, A classification of solutions of conformally invariant fourth order equations in $\mathbb{R}^{n}$, Comm. Math. Helv 73 (1998), 206-231. 
[18] L. Martinazzi, Classification of solutions to the higher order Liouville's equation on $\mathbb{R}^{2 m}$, Math. Z. 263 (2009), 307-329.

[19] L. Martinazzi, Conformal metrics on $\mathbb{R}^{n}$ with constant $Q$-curvature and large volume, Ann. Inst. Henri Poincaré (C) 30 (2013), 969-982.

[20] J. Prajapat, G. Tarantello, On a class of elliptic problems in $\mathbb{R}^{2}$ : symmetry and uniqueness results, Proc. Royal Soc. Edinburgh 131A (2001), 967-985.

[21] M. Troyanov, Prescribing curvature on compact surfaces with conical singularities, Trans. Am. Math. Soc. 324 (1991) 793-821.

[22] J. WeI, X-W. Xu, Classification of solutions of higher order conformally invariant equations, Math. Ann 313 (1999), 207-228.

[23] J. WeI, D. YE, Nonradial solutions for a conformally invariant fourth order equation in $\mathbb{R}^{3}$, Calc. Var. Partial Differential Equations 32 (2008), no. 3, 373-386.

[24] X. XU, Uniqueness and non-existence theorems for conformally invariant equations, J. Funct. Anal. 222 (2005), 1-28. 